\title{
INTERACTION BETWEEN EDGE DISLOCATION AND INHOMOGENEITY OF AN ARBITRARY SHAPE AND PROPERTIES UNDER COUPLED THERMOMECHANICAL STRAINS
}

\author{
YANG SUN \\ Xi'an University of Technology, Department of Engineering Mechanics, Xi'an, China \\ e-mail: ysun@xaut.edu.cn \\ Ang Li, LAN SHI, MABAO LiU \\ Xi'an Jiaotong University, State Key Laboratory for Strength and Vibration of Mechanical Structures, Xi'an, China \\ e-mail:mliu@mail.xjtu.edu.cn
}

In this work, a general approximate solution for the configurational force between edge dislocation and inhomogeneity of an arbitrary shape and properties with coupled thermomechanical loads was developed on the basis of the Eshelby equivalent inclusion theory. The effect of temperature-dependent elastic properties, thermal expansion coefficient and yield strength on the configurational forces was analyzed. Furthermore, the configurational force considered to be the driving force for dislocation migration was innovatively used to investigate the interaction mechanism between graphene and internal defects of a metal.

Keywords: thermomechanical strain, edge dislocation, inhomogeneity

\section{Introduction}

Metal matrix composites (MMCs) are widely used in aerospace, automotive, and electronics fields due to their excellent mechanical and physical properties. Generally, metal matrix composites are subjected to high temperature during manufacturing and service. In a thermomechanical coupling environment, it is inevitably for dislocation to occur. Subsequently, it is necessary to understand the relation of the dislocation mobility and the micro failure of MMCs. Currently, it is generally believed that the micro failure of MMCs is strongly influenced by various elastic inhomogeneity along with non-elastic inhomogeneity including a void, plastic zone of crack tip.

Though some approximate methods of interaction between inhomogeneity of an arbitrary shape and dislocation have been established based on the Eshelby equivalent inclusion theory (Li et al., 2011; Zhang et al., 2013), these investigations were mainly concentrated on the mechanical strain. In many engineering cases, however, MMCs are often subjected to thermal strain (Wei et al., 2016; Ebrahimi et al., 2016). Unfortunately, no researches have been reported on the interaction of edge dislocation with inhomogeneity under coupled thermomechanical strains so far.

In the Eshelby equivalent inclusion theory, thermal strain can be regarded as additional transformation strain to investigate the mismatch between the matrix and inhomogeneity. Withers et al. (1989) assumed isotropic thermal expansion of the matrix and the reinforcement, and studied the thermal residual stress field induced by difference of the thermal expansion coefficient between $\mathrm{SiC}$ short fibers and an aluminum matrix utilizing the Eshelby theory. Peng et al. (2015) investigated inclusion-crack interaction with coupled mechanical and thermal strains, and obtained a closed-form solution for the stress intensity factor based on the Eshelby equivalent inclusion theory and transformation toughening theory. Bennett et al. (2018) studied thermoelasticity of porous bonded-particle assemblies considering damage at inter-particle interfaces. 
They developed a modified self-consistent homogenization for particles of an ellipsoidal shape with anisotropic thermal and elastic expansion properties. For the elastic inhomogeneity, the equivalent transformation strain can be achieved from the Eshelby equivalent inclusion theory. Nevertheless, it is quite difficult to obtain the transformation strain of non-elastic inhomogeneity by the existing methods under coupled thermomechanical strains.

When the configuration (size, shape or position) of material defects varies, the free energy of the material will change as well. The driving force related to the variation of the configuration can be designated as a configurational force (CF). The CF was firstly introduced by Eshelby (1951). The configurational mechanics based on the material space can provide a new way to solve defects of composites under complex loads. The behavior of crack deflection/penetration in composites was investigated by the configurational force theory (Sun et al., 2018). Lv et al. (2017) adopted the theory to analyze the influence of growth of the thermal barrier coating layer on the crack-tip driving force. Baxevanakis and Georgiadis (2019) discussed the energy release rate and the configurational force exerted on a climb dislocation dipole, and found that the energy release rate was significantly influenced by the defect distance and the characteristic material length. A damage-based temperature-dependent model for ductile fracture was successfully established based on the concept of the configurational force in literature (Van Goethem and Areias, 2012).

In this paper, an alternative method was proposed to determine the transformation strain in various inhomogeneity under coupled thermomechanical loads based on the stress equivalence principle of the Eshelby inclusion theory (Eshelby, 1951) and an approximate continuum theory for the interaction of dislocation with inhomogeneity ( $\mathrm{Li}$ et al., 2011; Zhang et al., 2013). Then, a general approximate solution for the configurational forces between edge dislocation and inhomogeneity with coupled thermomechanical strains was developed, in which the thermal expansion strains between the matrix and inhomogeneity were considered as eigenstrains. Moreover, the effect of temperature-dependent elastic properties, thermal expansion coefficient and yield strength on the configurational force was estimated for graphene reinforced metal composites.

\section{Model and formulation}

Figure 1 shows the physical problem of this study. Straight edge dislocation whose line coincides with the $z$-axis of a Cartesian coordinate system is located at the point $(0,0,0)$. The inhomogeneity of an arbitrary shape (domain $\Omega$ ) embedded in an infinite matrix is considered, which is subjected to the dislocation stress field and the eigenstrain.

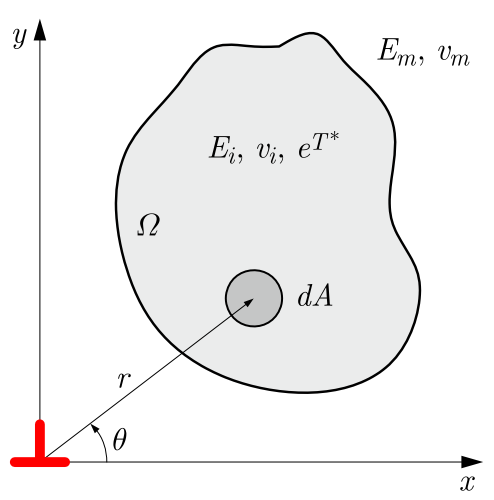

Fig. 1. Edge dislocation near inhomogeneity of an arbitrary shape 
The stress field in the infinite matrix with inhomogeneity can be obtained from the Eshelby inclusion theory (Li et al., 2011; Eshelby, 1951; Mura, 1987). Based on this theory, the stress is uniform in an inclusion, which can be expressed as

$$
\boldsymbol{\sigma}=\mathbf{C}_{m} \mathbf{e}
$$

or

$$
\boldsymbol{\sigma}=\mathbf{C}_{m}\left(\mathbf{e}^{C}+\mathbf{e}^{A}-\mathbf{e}^{\mathrm{T}}\right)
$$

where $\mathbf{C}_{m}$ is the elastic tensor of the matrix material; $\mathbf{e}$ is the elastic strain in the equivalent homogeneity; $\mathbf{e}^{A}$ is the combined strain field of the externally applied strain field and the dislocation field in the absence of the inclusion; $\mathbf{e}^{\mathrm{T}}$ is the transformation strain in the inclusion; $\mathbf{e}^{C}$ is the constrained strain, i.e.

$$
\mathbf{e}^{C}=\mathbf{S e}^{\mathrm{T}}
$$

Combining Eqs.(2.1)-(2.3), one can obtain

$$
\mathbf{e}^{\mathrm{T}}=(\mathbf{S}-\mathbf{I})^{-1} \varepsilon
$$

in which

$$
\varepsilon=\mathbf{e}-\mathbf{e}^{A}
$$

Thus, the transformation strain can be determined when the elastic strain $\mathbf{e}$ in the domain $\Omega$ is given. The common non-elastic inhomogeneity includes voids, plastic zones, etc. The elastic strain of the void is a zero tensor, and the elastic strain of the plastic zone can be obtained from the critical stress intensity factor ( $\mathrm{Li}$ et al., 2011). In addition, the transformation strain in the differential element $d A$ within the elastic inhomogeneity $\mathbf{e}^{\mathrm{T}}$ can be expressed as

$$
\mathbf{e}^{\mathrm{T}}=(1-\alpha)[(\alpha-1) \mathbf{S}+\mathbf{I}]^{-1} \mathbf{e}^{A}+\alpha[(\alpha-1) \mathbf{S}+\mathbf{I}]^{-1} \mathbf{e}^{* \mathrm{~T}}
$$

and $\mathbf{e}^{* \mathrm{~T}}$ is the thermal expansion strain, a typical eigenstrain (Mura, 1987), i.e.

$$
\mathbf{e}^{* \mathrm{~T}}=[(1+\nu) \Delta C T E \Delta T,(1+\nu) \Delta C T E \Delta T, 0]
$$

in which $\alpha=E_{i} / E_{m} ; E_{i}$ and $E_{m}$ are the modulus of inhomogenity and the metal matrix, respectively; $\triangle C T E$ is the difference of the thermal expansion coefficient between the inhomogenity and the metal matrix. For a differential element with a circular section inside the inclusion, the Eshelby tensor $\mathbf{S}$ for plane strain is given by (Mura, 1987)

$$
\mathbf{S}=\frac{1}{8(1-\nu)}\left[\begin{array}{ccc}
5-4 \nu & 4 \nu-1 & 0 \\
4 \nu-1 & 5-4 \nu & 0 \\
0 & 0 & 2(3-4 \nu)
\end{array}\right]
$$

where $\nu$ is Poisson's ratio of the matrix.

Per unit thickness, the elastic energy associated with the transformation between the dislocation and the differential element is given by (Li et al., 2011)

$$
d W=\sigma_{i j}^{d} e_{i j}^{\mathrm{T}} d A
$$

where $\sigma_{i j}^{d}$ is the stress field of dislocation. For edge dislocation, the nonzero stress components can be expressed by (Mura, 1987)

$$
\begin{aligned}
& \sigma_{11}^{d}=-\frac{\mu_{m} b}{2 \pi r(1-\nu)}\left(3 \sin \theta \cos ^{2} \theta+\sin ^{3} \theta\right) \quad \sigma_{22}^{d}=\frac{\mu_{m} b}{2 \pi r(1-\nu)}\left(\sin \theta \cos ^{2} \theta-\sin ^{3} \theta\right) \\
& \sigma_{12}^{d}=\frac{\mu_{m} b}{2 \pi r(1-\nu)}\left(\cos ^{3} \theta-\cos \theta \sin ^{2} \theta\right)
\end{aligned}
$$


where $\mu_{m}$ is the shear modulus of the matrix material, and $b$ is the Burgers vector of dislocation (Mura, 1987).

From Eq. (2.9), the total configurational force (CF) can be found in the absence of body forces

$$
d F=\frac{\partial(d W)}{\partial r}
$$

The total CFs acting on the dislocation along $x$ and $y$ directions can be respectively expressed as

$$
F_{x}=\int_{\Omega} \cos \theta d F \quad F_{y}=\int_{\Omega} \sin \theta d F
$$

The integrations are conducted on the whole domain $\Omega$ occupied by the inclusion. A positive (negative) value of $F$ corresponds to repulsion (attraction).

\section{Applications and discussion}

To verify the present solution, a graphene reinforced metal matrix composite is taken as an example. During manufacturing of the graphene/metal composite, various micro-defects (e.g., dislocations, voids, cracks and local plastic deformation zone) often appear in the metal matrix. In the absence of externally applied loads, the interaction forces of edge dislocation with a void and graphene (Shao et al., 2012) distributed in the grain boundary at different temperatures are calculated respectively, as shown in Fig. 2.

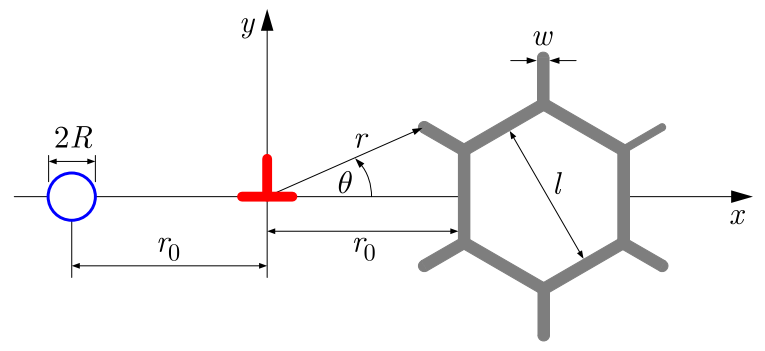

Fig. 2. Schematic of the interaction between dislocation, void and graphene

The inclusion of the void is equivalent to an elastic medium with zero stress based on the Eshelby theory, thereby the elastic strain $e_{i j}$ vanishes. In the absence of externally applied strain, the nonzero strains are (Mura, 1987)

$$
e_{11}^{A}=-\frac{b(1-2 \nu) \sin \theta}{4 \pi r(1-\nu)} \quad e_{22}^{A}=-\frac{b(1-2 \nu) \sin \theta}{4 \pi r(1-\nu)} \quad e_{12}^{A}=\frac{b \cos \theta}{4 \pi r(1-\nu)}
$$

for edge dislocation. Then, from Eq. (2.5), it can be obtained

$$
\varepsilon_{11}=-e_{11}^{A} \quad \varepsilon_{22}=-e_{22}^{A} \quad \varepsilon_{12}=-e_{12}^{A}
$$

Combining Eqs. (3.2), (2.4), (2.9) and (2.11), followed by numerical integration of Eq. (2.12), the total CFs between dislocation and the void are given by

$$
F_{\text {void-dislocation }}=\int_{\Omega} d F_{\text {void-dislocation }}
$$


By combining Eqs. (2.6), (2.7), (2.9) and (2.11), along with numerical integration of Eq. (2.12), the total CFs between dislocation and graphene can be expressed as

$$
F_{\text {graphene-dislocation }}=\int_{\Omega} d F_{\text {graphene-dislocation }}
$$
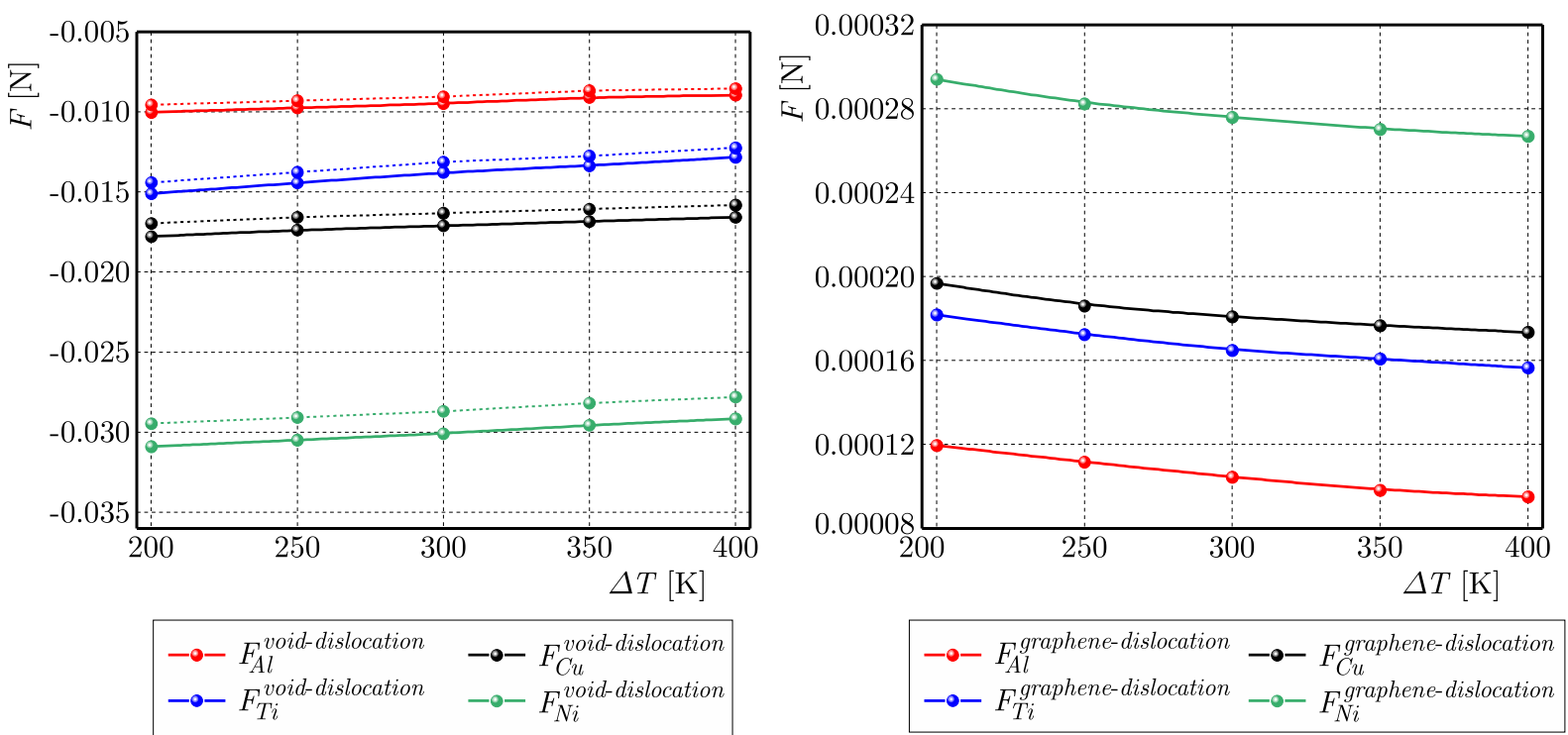

Fig. 3. Comparison of configurational forces at different temperatures: (a) edge dislocation interacting with a void for the present results and classical solutions; (b) edge dislocation interacting with graphene

Figure 3 shows the interaction between dislocation, graphene and a circular void of radius $R$ centered at $\left(-r_{0}, 0\right)$ (Fig. 2). It is assumed that the diameter $l$ of grains is approximately $200 \mathrm{~nm}$ in the metal matrix. The radius $R$, diameter $l$ and atomic layer thickness $w$ determine the integral domain $\Omega$. The mechanical parameters of the metal matrix at $200-400 \mathrm{~K}$ are given in Table 1. For the verification purpose, classical solutions are also shown in Fig. 3a. Compared with the classical solutions for edge dislocation interacting with the void (Dundurs and Mura, 1964), the estimates of the present method are in quite close agreement with the exact solutions, yielding the maximum difference below $5 \%$. On the other hand, the CF between dislocation and the void is an attractive force, which can explain dislocation annihilation when the void interacts with dislocation (Alquier et al., 2005). It should be noted that different metal matrices present different attractive forces, i.e., $F_{N i}>F_{C u}>F_{T i}>F_{A l}$, revealing a correlation with the elastic modulus. With an increase of $\Delta T$, the attraction force decreases progressively, suggesting that the probability of dislocation annihilation becomes smaller. As seen from Fig. 3b, the CF between dislocation and graphene presents a repulsion force, indicating that graphene hinders the movement of dislocations to the interface between metal and graphene. These results suggest that multiple dislocations pile up at the interface, which is well consistent with the experimental results (Kim, et al., 2013), thereby resulting in the strengthening effect of graphene/metal composites. The repulsion force decreases as $\Delta T$ increases, implying that the ability of graphene to block dislocations crossing the interface is weakened.

Figure 4 illustrates a schematic diagram of the interaction between dislocation, graphene and two circular voids of radius $R$ which are, respectively, centered at $\left(r_{0}, \alpha\right)$ and $\left(r_{0},-\alpha\right)$, and symmetric along the $x$ axis. As shown in Fig. 4, the total configurational forces are related to relative positions of voids, dislocation and graphene as well as Young's modulus of the metal matrix in Fig. 5. Keeping a fixed distance $r_{0}$ between voids and dislocation at $\Delta T=400 \mathrm{~K}$, a 
Table 1. Mechanical parameters used for model calculations (Ledbetter, 1982; Fisher and Renken, 1964; Gray, 1972; Callister and Rethwisch, 2014)

\begin{tabular}{|c|c|c|c|c|c|c|}
\hline & \multicolumn{5}{|c|}{ Temperature $[\mathrm{K}]$} \\
\hline & & 200 & 250 & 300 & 350 & 400 \\
\hline \multirow{2}{*}{ Copper } & $E$ [GPa] & 133 & 130 & 128 & 126 & 124 \\
\hline & $C T E\left[10^{-6} / \mathrm{K}\right]$ & 15.2 & 16.7 & 17.0 & 17.3 & 17.6 \\
\hline \multirow{2}{*}{ Aluminum } & $E[\mathrm{GPa}]$ & 75 & 73 & 71 & 68 & 67 \\
\hline & $C T E\left[10^{-6} / \mathrm{K}\right]$ & 20.2 & 22.0 & 23.6 & 24.1 & 24.9 \\
\hline \multirow{2}{*}{ Nickel } & $E[\mathrm{GPa}]$ & 231 & 228 & 225 & 221 & 218 \\
\hline & $C T E\left[10^{-6} / \mathrm{K}\right]$ & 11.2 & 12.2 & 12.8 & 13.5 & 14.1 \\
\hline \multirow{2}{*}{ Titanium } & $E[\mathrm{GPa}]$ & 113 & 108 & 103 & 100 & 96 \\
\hline & $C T E\left[10^{-6} / \mathrm{K}\right]$ & 7.4 & 8.1 & 8.6 & 9.1 & 9.4 \\
\hline
\end{tabular}

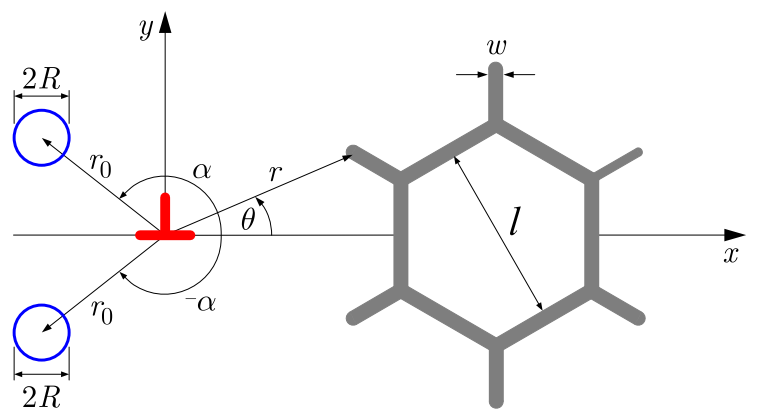

Fig. 4. Schematic of the interaction between dislocation, two voids and graphene

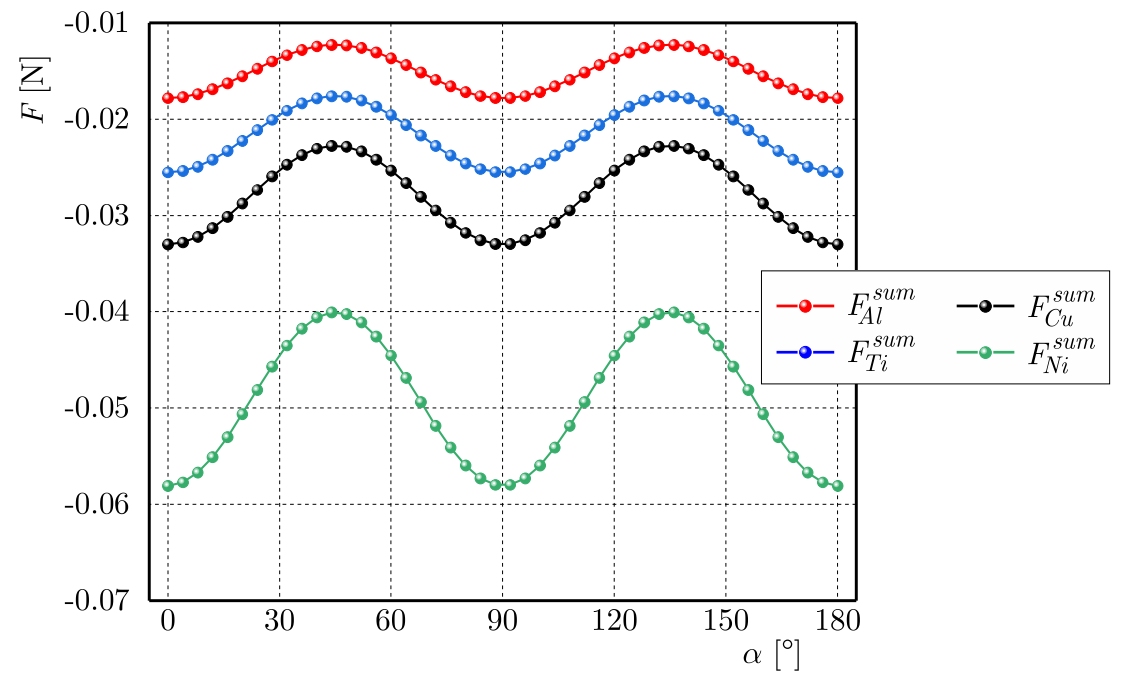

Fig. 5. Comparison of the total configurational forces between dislocation, graphene and two circular voids for different metal matrices at $\Delta T=400 \mathrm{~K}$

shielding effect is present on CF (attraction) when the angle $\alpha$ changes from $0^{\circ}$ to $45^{\circ}$ or from $90^{\circ}$ to $135^{\circ}$. Outside these regions, there is an amplification effect on CF. Furthermore, for edge dislocation, the configurational forces $F_{x}$ and $F_{y}$ are the glide and climb forces, respectively. The mobility of edge dislocation is solely dependent on $F_{x}$. Hence, further discussion is needed for $F_{x}$.

During the deformation process, the voids first appear in the vicinity of the metal/graphene interface. Under the action of continuous stress, the expanded voids give rise to formation of cracks. For most of metal materials, plastic zones will be generated at the tip of the cracks to prevent crack propagation (intrinsic toughening). When the size of the plastic zone is very small 
compared to the cracks, it is usually designated as the small scale yielding. At this case, the stress-strain field in the plastic zone of the crack tip is still controlled by the K-field. Therefore, linear elastic fracture mechanics is still applicable by an appropriate correction.

The combined strain field of the edge dislocation field (Mura, 1987) and the externally applied strain field of mode I/II crack tip (Li and Chen, 2002; Yang et al., 2004) are, respectively

$$
\begin{aligned}
& e_{11}^{A}=-\frac{b(1-2 \nu) \sin \theta}{4 \pi r(1-\nu)}+\frac{1+\nu}{E_{m}} \frac{K_{I}}{\sqrt{2 \pi r}} \cos \frac{\theta}{2}\left(1-2 \nu-\sin \frac{\theta}{2} \sin \frac{3 \theta}{2}\right) \\
& e_{22}^{A}=-\frac{b(1-2 \nu) \sin \theta}{4 \pi r(1-\nu)}+\frac{1+\nu}{E_{m}} \frac{K_{I}}{\sqrt{2 \pi r}} \cos \frac{\theta}{2}\left(1-2 \nu+\sin \frac{\theta}{2} \sin \frac{3 \theta}{2}\right) \\
& e_{12}^{A}=\frac{b \cos \theta}{4 \pi r(1-\nu)}+\frac{1+\nu}{E_{m}} \frac{K_{I}}{\sqrt{2 \pi r}} \cos \frac{\theta}{2} \sin \frac{\theta}{2} \cos \frac{3 \theta}{2}
\end{aligned}
$$

and

$$
\begin{aligned}
& e_{11}^{A}=-\frac{b(1-2 \nu) \sin \theta}{4 \pi r(1-\nu)}+\frac{1+\nu}{E_{m}} \frac{K_{I I}}{\sqrt{2 \pi r}} \sin \frac{\theta}{2}\left(-2+2 \nu-\cos \frac{\theta}{2} \cos \frac{3 \theta}{2}\right) \\
& e_{22}^{A}=-\frac{b(1-2 \nu) \sin \theta}{4 \pi r(1-\nu)}+\frac{1+\nu}{E_{m}} \frac{K_{I I}}{\sqrt{2 \pi r}} \sin \frac{\theta}{2}\left(2 \nu+\cos \frac{\theta}{2} \cos \frac{3 \theta}{2}\right) \\
& e_{12}^{A}=\frac{b \cos \theta}{4 \pi r(1-\nu)}+\frac{1+\nu}{E_{m}} \frac{K_{I I}}{\sqrt{2 \pi r}} \cos \frac{\theta}{2}\left(1-\sin \frac{\theta}{2} \sin \frac{3 \theta}{2}\right)
\end{aligned}
$$

under the plane strain condition.

Based on the stress field of mode I/II crack, the von Mises effective stress $\sigma_{e}$ is expressed as

$$
\sigma_{e}=\frac{K_{I}}{\sqrt{2 \pi r}} \cos \frac{\theta}{2} \sqrt{(1-2 \nu)^{2}+3 \sin ^{2} \frac{\theta}{2}}
$$

and

$$
\sigma_{e}=\frac{K_{I I}}{\sqrt{2 \pi r}} \sqrt{3 \cos ^{2} \frac{\theta}{2}\left(1-3 \sin ^{2} \frac{\theta}{2}\right)+4\left(\nu^{2}-\nu+1\right) \sin ^{2} \frac{\theta}{2}}
$$

respectively.

The above formulas can still be approximated at the elastic zone and plastic zone boundary, i.e., $r=r_{p}$, where the von Mises effective stress $\sigma_{e}$ is equal to the initial yield strength $\sigma_{y s}(\mathrm{Li}$ et al., 2011). Thus, the critical stress intensity factors of mode I/II crack are obtained

$$
\begin{aligned}
& K_{I}^{C}=\frac{\sigma_{y s} \sqrt{2 \pi r}}{\cos \frac{\theta}{2} \sqrt{(1-2 \nu)^{2}+3 \sin ^{2} \frac{\theta}{2}}} \\
& K_{I I}^{C}=\frac{\sigma_{y s} \sqrt{2 \pi r}}{\sqrt{3 \cos ^{2} \frac{\theta}{2}\left(1-3 \sin ^{2} \frac{\theta}{2}\right)+4\left(\nu^{2}-\nu+1\right) \sin ^{2} \frac{\theta}{2}}}
\end{aligned}
$$

In the case of small scale yielding and assumption of elastic-perfectly plastic, the elastic zone and plastic zone boundary of mode I/II crack yields

$$
r_{p}=\frac{K_{I}^{2}}{2 \pi \sigma_{y s}^{2}} \cos ^{2} \frac{\theta}{2}\left[(1-2 \nu)^{2}+3 \sin ^{2} \frac{\theta}{2}\right]
$$

and

$$
r_{p}=\frac{K_{I I}^{2}}{2 \pi \sigma_{y s}^{2}}\left[3 \cos ^{2} \frac{\theta}{2}\left(1-3 \sin ^{2} \frac{\theta}{2}\right)+4\left(\nu^{2}-\nu+1\right) \sin ^{2} \frac{\theta}{2}\right]
$$

respectively. 
The elastic strain $e_{i j}$ in the plastic zone can be obtained by substituting $K_{I}=K_{I}^{C}$ and $K_{I I}=K_{I I}^{C}$ in the second term on the right-hand side of Eqs. (3.5) and (3.6), respectively. From Eq. (2.5), the strains $\varepsilon$ are

$$
\begin{aligned}
& \varepsilon_{11}=\frac{b(1-2 \nu) \sin \theta}{4 \pi r(1-\nu)}+\frac{1+\nu}{E_{m}} \frac{K_{I}^{C}-K_{I}}{\sqrt{2 \pi r}} \cos \frac{\theta}{2}\left(1-2 \nu-\sin \frac{\theta}{2} \sin \frac{3 \theta}{2}\right) \\
& \varepsilon_{22}=\frac{b(1-2 \nu) \sin \theta}{4 \pi r(1-\nu)}+\frac{1+\nu}{E_{m}} \frac{K_{I}^{C}-K_{I}}{\sqrt{2 \pi r}} \cos \frac{\theta}{2}\left(1-2 \nu+\sin \frac{\theta}{2} \sin \frac{3 \theta}{2}\right) \\
& \varepsilon_{12}=-\frac{b \cos \theta}{4 \pi r(1-\nu)}+\frac{1+\nu}{E_{m}} \frac{K_{I}^{C}-K_{I}}{\sqrt{2 \pi r}} \cos \frac{\theta}{2} \sin \frac{\theta}{2} \cos \frac{3 \theta}{2}
\end{aligned}
$$

and

$$
\begin{aligned}
& \varepsilon_{11}=\frac{b(1-2 \nu) \sin \theta}{4 \pi r(1-\nu)}+\frac{1+\nu}{E_{m}} \frac{K_{I I}^{C}-K_{I I}}{\sqrt{2 \pi r}} \sin \frac{\theta}{2}\left(-2+2 \nu-\cos \frac{\theta}{2} \cos \frac{3 \theta}{2}\right) \\
& \varepsilon_{22}=\frac{b(1-2 \nu) \sin \theta}{4 \pi r(1-\nu)}+\frac{1+\nu}{E_{m}} \frac{K_{I I}^{C}-K_{I I}}{\sqrt{2 \pi r}} \sin \frac{\theta}{2}\left(2 \nu+\cos \frac{\theta}{2} \cos \frac{3 \theta}{2}\right) \\
& \varepsilon_{12}=-\frac{b \cos \theta}{4 \pi r(1-\nu)}+\frac{1+\nu}{E_{m}} \frac{K_{I I}^{C}-K_{I I}}{\sqrt{2 \pi r}} \cos \frac{\theta}{2}\left(1-\sin \frac{\theta}{2} \sin \frac{3 \theta}{2}\right)
\end{aligned}
$$

respectively.

Combining Eqs. (3.12), (3.13), (2.4), (2.9) and (2.11), followed by numerical integration of Eq. (2.12), the CFs between the dislocation and plastic zone of mode I/II crack tip can be obtained, respectively, as

$$
F_{x-I}^{\text {plastic zone-dislocation }}=\int_{\Omega} d F_{x-I}^{\text {plastic zone-dislocation }}
$$

and

$$
F_{x-I I}^{\text {plastic zone-dislocation }}=\int_{\Omega} d F_{x-I I}^{\text {plastic zone-dislocation }}
$$

respectively. The integrations are conducted on the whole domain $\Omega$ occupied by the plastic zone. To eliminate strain singularity in Eqs. (3.12) and (3.13), a range of $0.0005 r_{p} \leqslant r \leqslant r_{p}$ is defined.

Under the total strain field of edge dislocation field and the applied strain field of mode I/II crack tip, combining Eqs. (2.6), (2.7), (2.9) and (2.11), followed by numerical integration of Eq. (2.12), the CFs between dislocation and graphene can be obtained as

$$
F_{x-I}^{\text {graphene-dislocation }}=\int_{\Omega} d F_{x-I}^{\text {graphene-dislocation }}
$$

and

$$
F_{x-I I}^{\text {graphene-dislocation }}=\int_{\Omega} d F_{x-I I}^{\text {graphene-dislocation }}
$$

respectively.

The yield strength $\sigma_{y s}$ at the temperature $T$ is (Li et al., 2016)

$$
\sigma_{y s}=\sqrt{\frac{E(T)}{E\left(T_{0}\right)}\left(1-\frac{\int_{T_{0}}^{T} C_{p}(T) d T}{\int_{T_{0}}^{T_{m}} C_{p}(T) d T}\right)} \sigma_{y s 0}
$$


where $E_{T}$ is Young's modulus at the temperature $T, T_{0}$ is an arbitrary reference temperature, $T_{m}$ is the melting point of the material and $\sigma_{y s 0}$ is the yield strength at the temperature $T_{0}$. In this work, the effect of temperature dependent Poisson's ratio $\nu$ on $\sigma_{y s}$ is neglected $(\nu=0.3)$.

$C_{p}(T)$ is the specific heat capacity for constant pressure and temperature $T$, expressed as (Ye, 2002)

$$
C_{p}(T)=A_{1}+A_{2} \cdot 10^{-3} T+A_{3} \cdot 10^{5} T^{-2}+A_{4} \cdot 10^{-6} T^{2}+A_{5} \cdot 10^{8} T^{-3}
$$

Taking the room temperature as the reference temperature, the yield strengths for titanium, nickel, copper and aluminum are $170 \mathrm{MPa}$ (annealed), $148 \mathrm{MPa}$ (annealed), $69 \mathrm{MPa}$ (hot rolled), $34 \mathrm{MPa}$ (annealed), respectively (Callister and Rethwisch, 2014). Substituting Eq. (3.19) into (3.18), $\sigma_{y s}$ can be obtained, which is shown in Fig. 6.

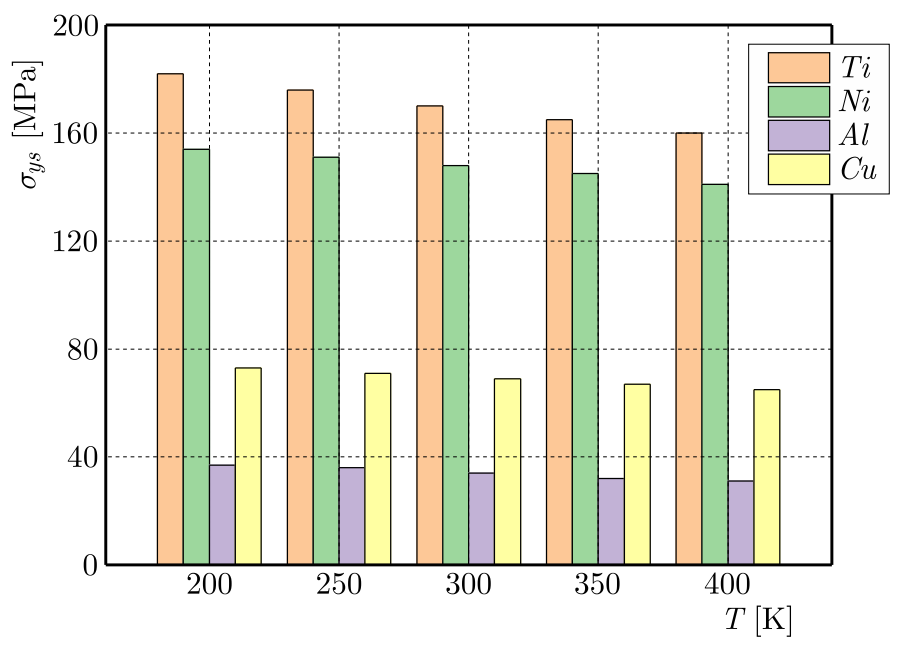

Fig. 6. The yield strength for $\mathrm{Ti}, \mathrm{Ni}, \mathrm{Al}, \mathrm{Cu}$ matrix at different temperatures

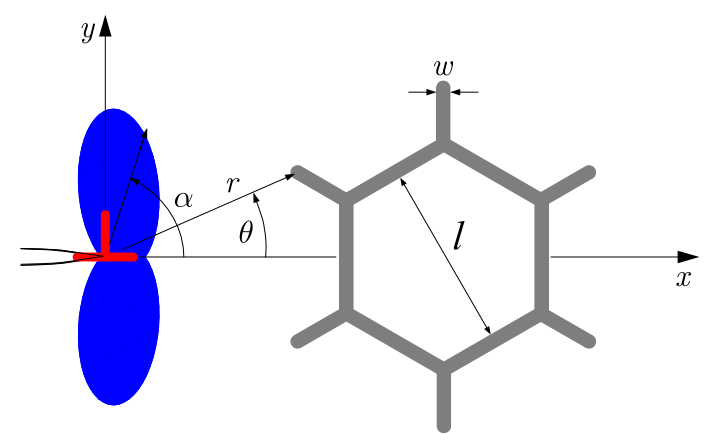

Fig. 7. Schematic of the interaction between dislocation, graphene and the plastic zone of mode I crack tip

In the present work, the image force acting on edge dislocation induced by the free surface of the crack and the slip force acting on edge dislocation induced by the crack-tip stress field are not considered. For simplicity, dislocation is placed at the crack tip utilizing the method reported by Zhang et al. (2013). The diagrams in Fig. 7 present the interaction between dislocation, graphene and the plastic zone of mode I crack tip. As shown in Fig. 8, under the combined strain field, the $\mathrm{CF}\left(F_{\text {glide }}\right)$ between the plastic zone of mode I crack tip and dislocation exhibits as repulsion, which decreases with an increase of $\Delta T$, implying that the ability of dislocation deviating from the plastic zone becomes weakened. The more dislocations in the plastic zone, the stronger plasticity and toughness of composites. $F_{\text {glide }}$ between graphene and dislocation is attractive, 
and increases as $\Delta T$ increases. Besides, the total $F_{\text {glide }}$ (repulsive force) between mode I plastic zone, graphene and dislocation decreases with an increase of $\Delta T$, and $F_{T i}>F_{N i}>F_{C u}>F_{A l}$ at the same $\Delta T$, which is related to the yield strength.

(a)

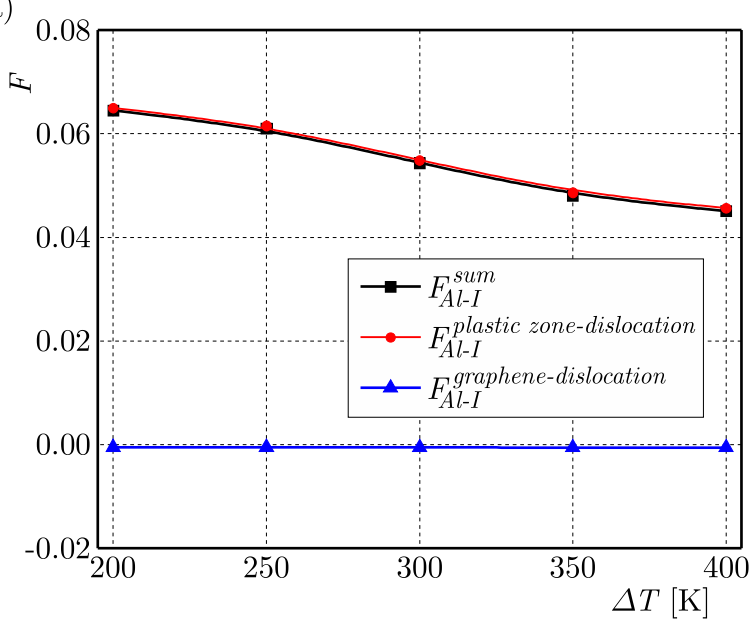

(b)

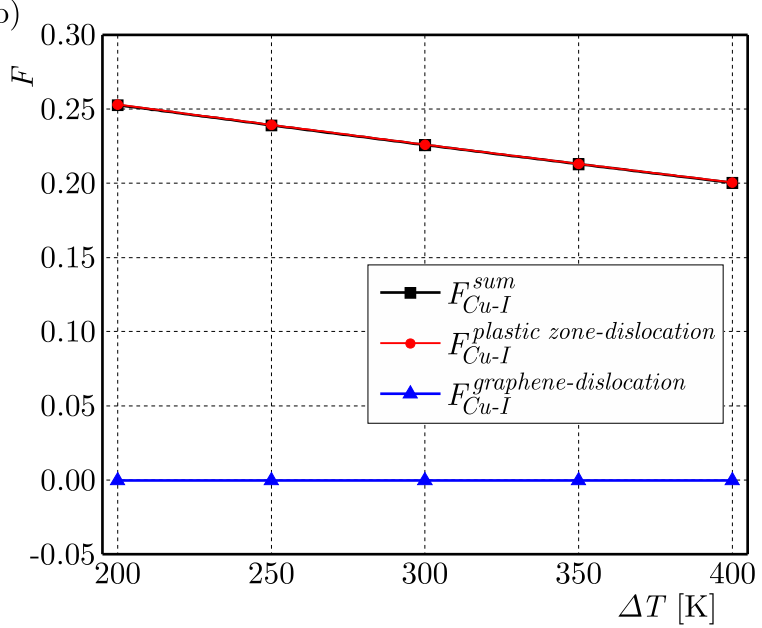

(c)

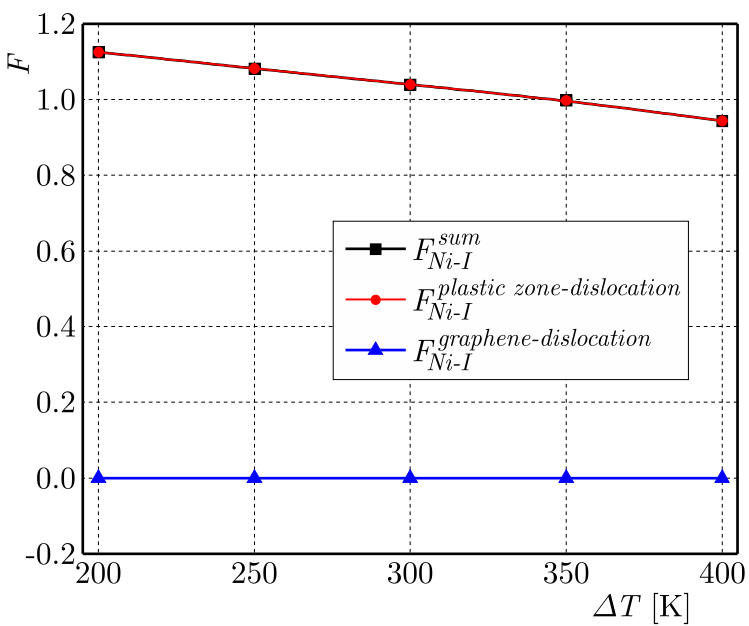

(d)

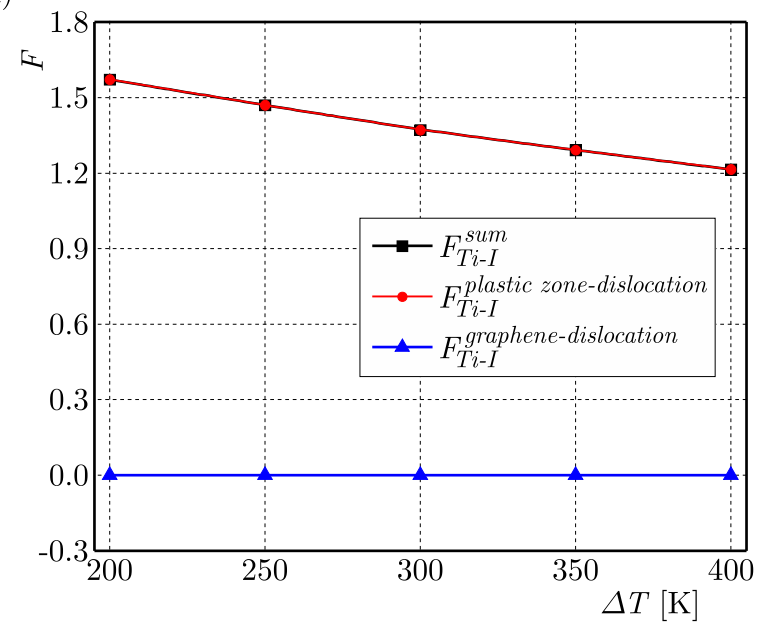

Fig. 8. Comparison of the configurational forces $F_{\text {glide }}$ (in unit of $\mu b /[\pi(1-2 \nu)]$ ) between dislocation, graphene and the plastic zone of mode I crack tip for different $\Delta T$ at $K_{I}=200 \mathrm{MPa} \sqrt{\mathrm{m}}$, $b=1 \cdot 10^{-10} \mathrm{~m}$ : (a) Al matrix, (b) Cu matrix, (c) Ni matrix, (d) Ti matrix

Figure 9 displays a schematic diagram of the interaction between dislocation, graphene and the plastic zone of mode II crack tip. Under the combined strain field, $F_{\text {glide }}$ between dislocation and graphene is a repulsive force for $\mathrm{Al}, \mathrm{Cu}, \mathrm{Ti}$ and $\mathrm{Ni}$ matrix. With an increase of $\Delta T$, an amplification effect is present on $F_{\text {glide }}$. On the other hand, $F_{\text {glide }}$ between the plastic zone of mode II crack tip and dislocation is attractive, which decreases as $\Delta T$ increases for $\mathrm{Al}, \mathrm{Cu}$ and $\mathrm{Ni}$ matrices, suggesting that the attraction of the plastic zone to dislocations is attenuated. However, the effect of temperature on $F_{\text {glide }}$ between the plastic zone and dislocation is reversed for the Ti matrix. For different metal matrices, it has a remarkable difference for the total interaction forces of mode II plastic zone, graphene and dislocation. The total $F_{\text {glide }}$ becomes repulsive, and increases as $\Delta T$ increases for $\mathrm{Al}$ matrix, whereas the total $F_{\text {glide }}$ displays an attractive force, and decreases with an increase of $\Delta T$ for $\mathrm{Ni}$ and $\mathrm{Cu}$ matrices. Nevertheless, there is an opposite trend for Ti matrix. 


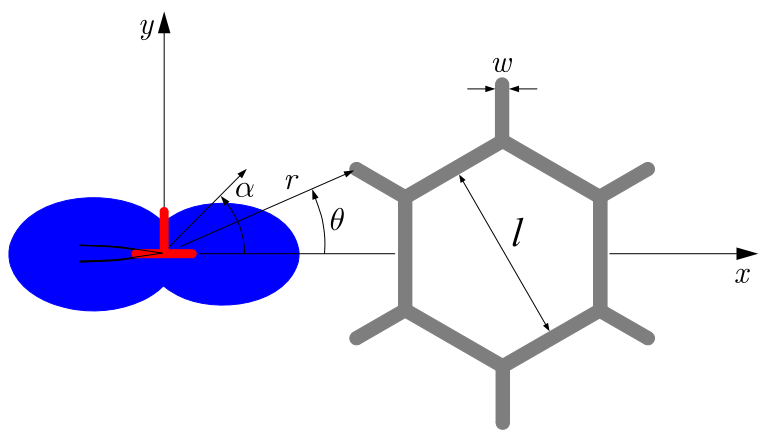

Fig. 9. Schematic of the interaction between dislocation, graphene and the plastic zone of mode II crack tip

(a)

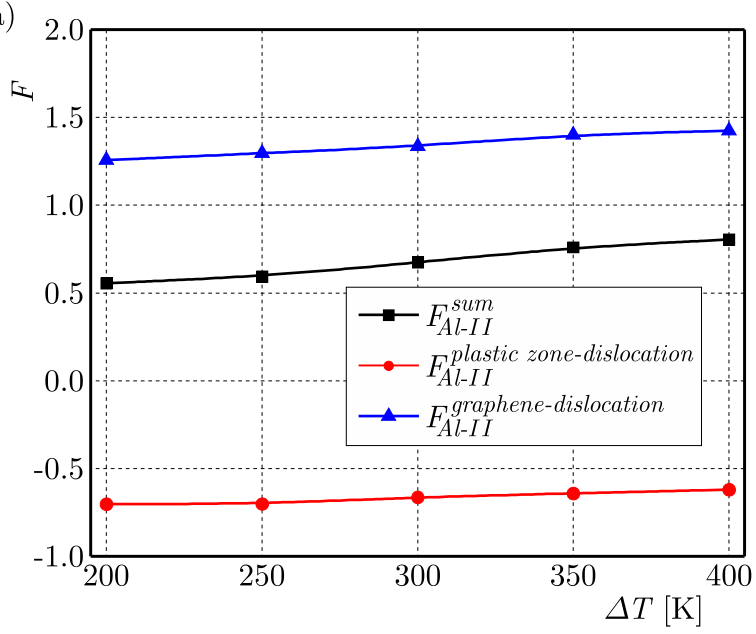

(c)

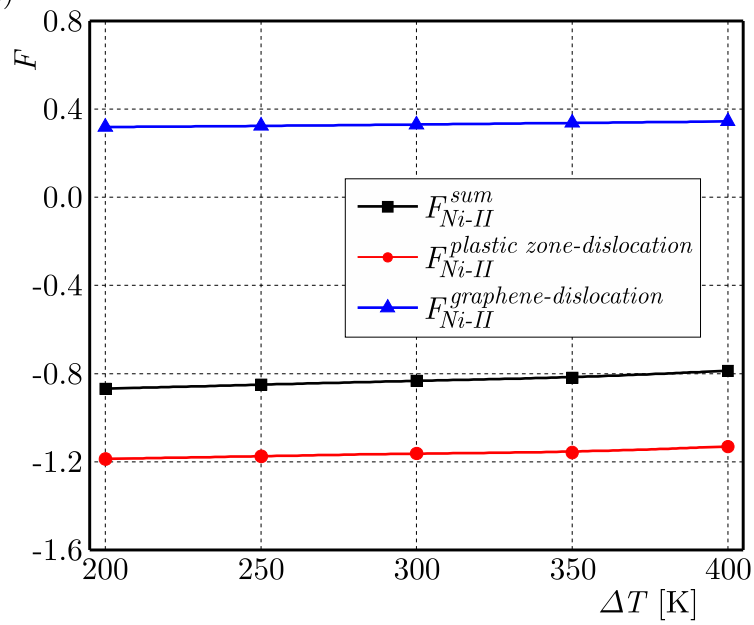

(b)

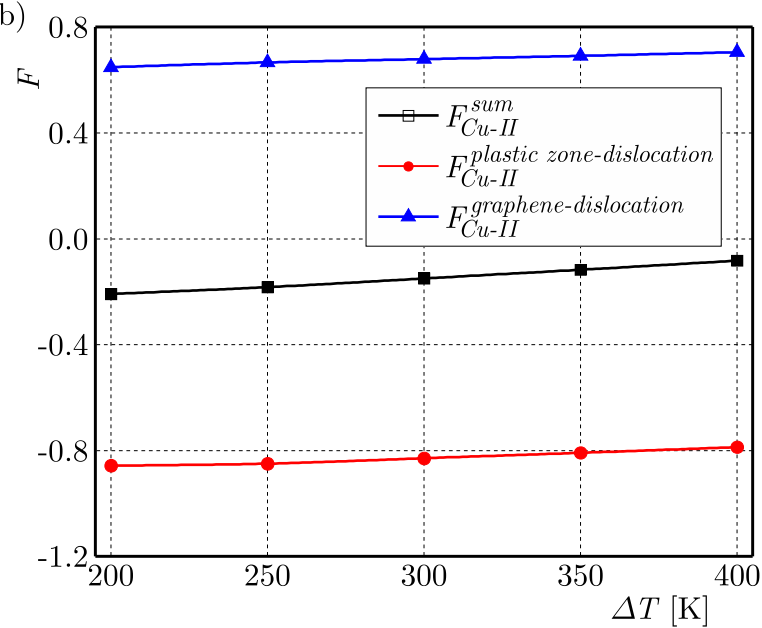

(d)

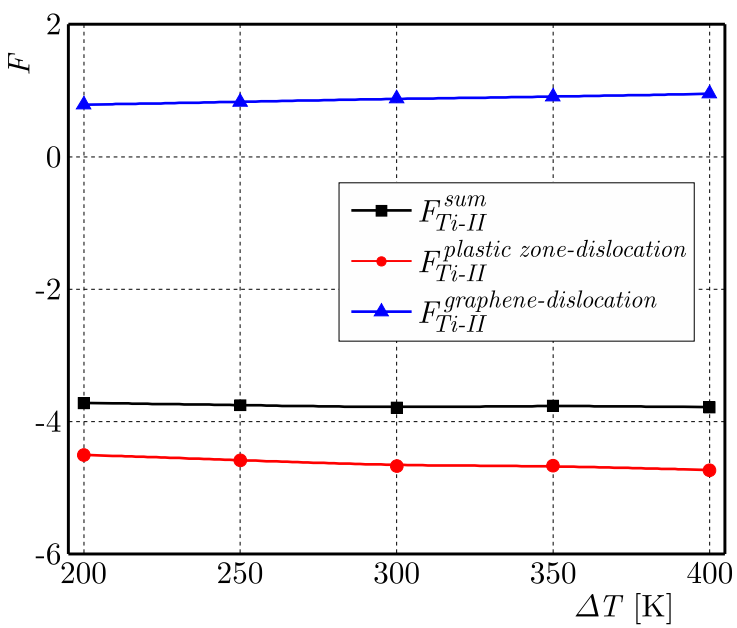

Fig. 10. Comparison of the configurational forces $F_{\text {glide }}$ (in unit of $\mu b /[\pi(1-2 \nu)]$ ) between dislocation, graphene and the plastic zone of mode II crack tip for different $\Delta T$ at $K_{I I}=200 \mathrm{MPa} \sqrt{\mathrm{m}}$, $b=1 \cdot 10^{-10} \mathrm{~m}$ : (a) Al matrix, (b) Cu matrix, (c) Ni matrix, (d) Ti matrix

\section{Conclusions}

In this study, an inhomogeneous inclusion was transformed into a homogenous one with an equivalent transformation strain based on the Eshelby theory. The interaction between edge dislocation and inhomogeneity was evaluated by the configuration force theory. A general solution for the configuration force was developed to investigate the interaction between edge 
dislocation and inhomogeneity of an arbitrary shape under coupled thermomechanical strains. The presented solutions can provide a novel way to describe the interaction mechanism between graphene and internal defects of metals, which is in good accordance with the experimental results. More importantly, the present approach with the basic equation in the form of an integral can be applicable to inhomogeneity with an arbitrary shape and properties.

\section{Acknowledgments}

The authors would like to acknowledge the financial support by the National Natural Science Foundation of China (11972284), Scientific research project of Shaanxi Education Department (20JK0799), Shaanxi National Science Foundation (2018JQ1029) and the Doctoral Initial Research Foundation of Xi'an University of Technology (256081810).

\section{References}

1. Alquier D., Bongiorno C., Rocchforte F., Raineri V., 2005, Interaction between dislocations and He-implantation-induced voids in GaN epitaxial layers, Applied Physics Letters, 86, 211911

2. Baxevanakis K.P., Georgiadis H.G., 2019, A displacement-based formulation for interaction problems between cracks and dislocation dipoles in couple-stress elasticity, International Journal of Solids and Structures, 159, 1-20

3. Bennett K.C., Luscher D.J., Buechler M.A., Yeager J.D., 2018, A micromechanical framework and modified self-consistent homogenization scheme for the thermoelasticity of porous bonded-particle assemblies, International Journal of Solids and Structures, 139, 224-237

4. Callister JR W.D., Rethwisch D.G., 2014, Materials Science and Engineering: An Introduction, 9rd Ed., John Wiley \& Sons Inc.

5. Dundurs J., Mura T., 1964, Interaction between an edge dislocation and a circular inclusion, Journal of the Mechanics and Physics of Solids, 12, 3, 177-189

6. Ebrahimi F., Barati M.R., Dabbagh A., 2016, A nonlocal strain gradient theory for wave propagation analysis in temperature-dependent inhomogeneous nanoplates, International Journal of Engineering Science, 107, 169-182

7. Eshelby J.D., 1951, The force on an elastic singularity, Pilosophical Transactions of the Royal Society of London Series A: Mathematical and Physical Sciences, 244, 877, 87-112

8. Fisher E.S., Renken C.J., 1964, Single-crystal elastic moduli and the hcp-bcc transformation in Ti, Zr, and Hf, Physical Review, 135, 482-494

9. Gray D.E., 1972, American Institute of Physics Handbook, 3rd Ed., McGraw-Hill Inc.

10. Kim Y., Lee J., Yeom M.S., Shin J.W., Kim H., Cui Y., Kysar J.W., Hone J., Jung Y., JEON S., HAN S.M., 2013, Strengthening effect of single-atomic-layer graphene in metal-graphene nanolayered composites, Nature Materials, 4, 2114

11. Ledbetter H.M., 1982, Temperature behaviour of Young's moduli of forty engineering alloys, Cryogenics, 22, 653-656

12. Li W., Zhang X., Kou H., Wang R., Fang D., 2016, Theoretical prediction of temperature dependent yield strength for metallic materials, International Journal of Mechanical Sciences, 105, $273-278$

13. Li Z., Chen Q., 2002, Crack-inclusion interaction for mode I crack analyzed by Eshelby equivalent inclusion method, International Journal of Fracture, 118, 1, 29-40

14. Li Z., Li Y., Sun J., Feng X.Q., 2011, An approximate continuum theory for interaction between dislocation and inhomogeneity of any shape and properties, Journal of Applied Physics, 109, 11, 113529 
15. Lv J.N., FAN X.L., Li Q., 2017, The impact of the growth of thermally grown oxide layer on the propagation of surface cracks within thermal barrier coatings, Surface and Coatings Technology, 309, 1033-1044

16. Mura T., 1987, Micromechanics of Defects in Solids, 2nd Ed., Martinus Nijhoff Publishers

17. Peng B., Feng M., FAn J., 2015, Study on the crack-inclusion interaction with coupled mechanical and thermal strains, Theoretical and Applied Fracture Mechanics, 75, 39-43

18. Shao T., Wen B., Melnik R., Yao S., Kawazoe Y., Tian Y.J., 2012, Temperature dependent elastic constants and ultimate strength of graphene and graphyne, The Journal of Chemical Physics, 137, 194901

19. Sun Y., Yu X., Jia W., Wang X., LiU M., 2018, The interaction of the mode II crack with an inhomogeneity undergoing a stress-free transformation strain, Acta Mechanica, 229, 3, 1311-1320

20. Van Goethem N., Areias P., 2012, A damage-based temperature-dependent model for ductile fracture with finite strains and configurational forces, International Journal of Fracture, 178, 215-232

21. Wei Y., Zhang L., Au F.T.K., Li J., Tsang N.C.M., 2016, Thermal creep and relaxation of prestressing steel, Construction and Building Materials, 128, 118-127

22. Withers P.J., Stobbs W.M., Pedersen O.B., 1989, The application of the Eshelby method of internal stress determination to short fiber metal matrix composites, Acta Metallurgica, 37, 11, 3061-3084

23. Yang L.H., Chen Q., Li Z.H., 2004, Crack-inclusion interaction for mode II crack analyzed by Eshelby equivalent inclusion method, Engineering Fracture Mechanics, 71, 1421-1433

24. YE D.L., 2002, Practical Handbook of Thermodynamic Data for Inorganic Compounds, 2nd Ed., Metallurgy Industry Publishing House

25. Zhang C.L., Li S., Li Z,H., 2013, The interaction of an edge dislocation with an inhomogeneity of arbitrary shape in an applied stress field, Mechanics Research Communications, 48, 19-23 